\title{
POLIGINIA Y FECUNDIDAD EN LOS SHIPIBO DE LA Amazonía Peruana
}

\author{
Warren M. Hern
}

En este tercer y final estudio, el autor se propone probar la hipótesis especifica de que la poliginia, en los shipibo, limita la fecundidad de las mujeres a nivel individual y comunal mediantela abstinencia post-partum y la prolongación de los intervalos entre cada nacimiento que, en una unión polígina, son más largos. Es de esta manera cómo los efectos de la modernización no necesariamente se asocian a una fecundidad más baja sino que, inclusive, la incrementa en la medida en que rompe sus prácticas culturales tradicionales. Sin embargo, el mismo autor nos previene de generalizar los resultados de su estudio hacia toda la etnia shipibo o poblaciones indígenas en general.

In this third and final article, the author proposes to prove the specific hypothesis that polygyny, among the Shipibo, limits the fertility of women on the individual and communal levels through post-partum sexual abstinence and the lengthening of the intervals between each birth which, in a polygynous union, are longer. It is in this way that the effects of modernization are not necessarily related to lower fertility, but rather it is increased as the traditional cultural practices are transgressed. However, the author himself warns against generalizing from the results of this study to include the entire Shipibo ethnic group or indigenous populations in general. 


\section{INTRODUCCION}

En este informe describo las características de fecundidad de una tribu indígena Amazónica que manifiesta la fecundidad documentada más alta de cualquier agrupación humana, con una tasa bruta de reproducción de 4.933 en 1964-69 (Hern, 1977). Esta tribu, los Shipibo, está experimentando un cambio cultural rápido que incluye una disminución en la prevalencia de poliginia. Vamos a probar la hipótesis específica que la poliginia limita ambas la fecundidad de las mujeres individuales y de la comunidad por los mecanismos de abstinencia sexual post-partum e intervalos más largos entre los nacimientos. Los intervalos entre nacimientos de las mujeres en uniones políginas son más largos, y la fecundidad de estas mujeres es más baja. La tasa de fecundidad global de la comunidad es asociada negativamente con la proporción de intervalos entre nacimientos que ocurren en uniones políginas.

La disrupción de las tradiciones de control cultural sobre la fecundidad puede proveer una explicación de fecundidad aumentada en algunas agrupaciones étnicas que están experimentando cambios culturales acelerados ( Nag, 1980). Esto puede ser causado por una disminución en la duración de lactancia y por una disminución en la duración de abstinencia sexual después del parto observada por las mujeres. Esta es generalmente la verdad en Africa (Dorjahn, 1958; Cald well y Caldwell, 1977; Aborampah, 1987; Chojnacka, 1980; Cleveland, 1987; Isaac, 1980; Handwerker, 1987; Page y Lesthaeghe, 1981), aunque observaciones de lo contrario han sido publicadas (Sembajwe, 1979; Olusanya, 1971). La poliginía ha sido frecuentemente asociada con periodos prolongados de abstinencia sexual post-partum por las mujeres, y esta costumbre lógicamente debe resultar en intervalos prolongados entre los nacimientos (Whiting, 1964; Schoenmackers, et al, 1981). Un error común en la mayoría de los estudios es la falta de información sobre la fecundidad individual que relaciona el estado polígino con la duración de intervalos entre los nacimientos. Solamente en algunos estudios han hecho comparaciones de las éxperiencias de fecundidad de las mujeres políginas con las mujeres monógamas dentro de la misma sociedad. Esto es porque los cambios culturales que afectan la fecundidad ocurren más frecuentemente en las sociedades tribales o campesinas sin documentación adecuada, y la información sobre estas sociedades es recogida mayormente por antropólogos que no están capacitados para recoger datos demográficos (Petersen, 1975; Caldwell, Caldwell, y Caldwell, 1987). Una excepción notable es el estudio de los Kipsigis de Borgerhoff Mulder, los cuales muestran diferencias importantes entre la fecundidad de mujeres políginas y las monógamas (Borgerhoff Mulder, 1989). Además, Garenne y van de Walle 
(1989), en sus estudios de los Sereer, encontraron que la fecundidad es más baja en las mujeres políginas. Fuera de Africa, estudios de agrupaciones tan distintos como las de los Mormones, los moradores de Bangladesh, y las tribus de Nueva Guinea han mostrado que la poliginia disminuye la fecundidad de las mujeres (Smith y Kunz, 1976; Anderton y Emigh, 1989; Bowers, 1971; van Arsdale, 1978; Wood, Johnson, y Campbell, 1985; Shaikh, Aziz, y Chowdhury, 1987).

La poliginia es común en sociedades amazónicas deSur América (Siskind, 1973; Jackson, 1983), pero su prevalencia e impacto en la fecundidad no ha sido bien documentado. Casi todas las tasas de poliginia en Sur América están basadas en el número de hombres en uniones políginas, y una tasa del 27 por ciento ha sido hallada en familias de los Cashinahua junto con una "política poblacional" de la tribu que aumenta la fecundidad (Johnston, Kensinger, et al, 1969; Johnston y Kensinger, 1971). Chagnon $(1977,1979)$ ha notado tasas de poliginia tan altas como del 50 por ciento en los Yanomama, que observan abstinencia sexual post partum muy severa y que como consecuencia experimentan intervalos entre los nacimientos prolongados (promedio: 3.4 años). Los intervalos entre los nacimientos de los Xavante, que también observan poliginia sororal, son igualmente prolongados, y la fecundidad es baja, pero las observaciones pueden ser debido al infanticidio en las dos tribus (Neel y Chagnon, 1968; Neel y Salzano, 1970). Early y Peters (1990) han atribuido los intervalos prolongados en los Mucajai Yanomama a la duración prolongada de lactancia y aborto inducido.

En mis propios estudios iniciales de los Shipibo, noté una tasa de poliginia por casa de 7.1 por ciento, con 9.8 por ciento en todas las mujeres en edad de reproducción (edad 15+ años) en uniones políginas. Según la información local, la prevalencia de poliginia estaba disminuyendo. Los anticonceptivos herbales, utilizados por muchas mujeres Shipibo, alcanzaron su efectividad putativa por consecuencia de ser asociado con la observación simultánea de abstinencia sexual (Hern, 1976).

La poliginia no afecta la misma fecundidad de la mujer, más bien es una variable intermediaria que puede afectarla por consecuencia de la abstinencia sexual post-partum y disminuida frecuencia de coito (Bongaarts, 1978; Bongaarts y Potter, 1983, Nag, 1980). Otros factores anexos que afectan a la fecundidad son infecundabilidad post-natal causada por amenorrea anovulatoria debido a la supresión de lactancia, que parece ser importante en los IKung (Konner y Worthman, 1980; Lee, 1980). 
La lactancia es un método incierto para suprimir la ovulación, pero probablemente tiene más efectividad en las sociedades tribales en que el infante puede mamar frecuentemente en intervalos intermitentes, como hacen los Shipibo.

En este estudio, el estado de poliginia se usa como una variable substitutoria del control (Greenland y Sanders, 1980) para la abstinencia sexual post-partum, que se asume es una de las variables independientes más importantes que afectan la fecundidad:

\section{ANTECEDENTES}

Cerca de 25,000 miembros de la etnia Shipibo-Conibo viven en la cuenca del Kío Ucayali en la Amazonía Peruana alreredor de la ciudad de Pucallpa. Han mantenido su identidad cultural a pesar de más de 300 años de contacto con la cultura occidental. Son horticultores que dependen de la caza, la recolección, y la pesca para el autoconsumo y la satisfacción de sus necesidades como la obtención de la proteína, pero están entrando cada vez más en la economía nacional por medio del cultivo de arroz (Behrens, 1984; 1989)

Los Shipibo son adherentes a la matrilocalidad y, probablèmente, a la matrilinealidad (Abelove, 1978). La poliginia sororal es la forma más comun y preferida de la poliginia. Un hombre puede recibir como segunda esposa cualquier mujer que la primera esposa clasifica como hermana, pero en la práctica actual solamente las hermanas menores de las primeras esposas son recibidas. Típicamente, cada esposa tiene su propio hogar o, por lo menos, su propia candela, generalmente adyacente a las candelas de las otras esposas.

Durante los últimos 40 años, los Shipibo han experimentado un cambio cultural rápido y también han tenido un mayor contacto con la cultura occidental por medio del sistema económico y la fundación de un hospital particular, el Hospital Amazonico "Albert Schweitzer" en 1962. Mi primera experiencia de trabajo con los Shipibo fue cuando era un estudiante de medicina del tercer año en 1964. En esta época, trabajé en el Hospital Amazónico por un plazo de tres meses y después hice una visita de reconocimiento y censo inicial en una comunidad Shipibo por otros tres meses. Este estudio fue seguido por trabajos de campo incluyendo censos y estudios de salud en 1969, 1974, y 1979 también con el estudio actual por un total de 20 meses. 
$\checkmark$ Uno de las observaciones más impresionantes es la disminución en la prevalencia de poliginia sororal (Hern, 1988). Los misioneros han desalentado la poliginia desde sus primeros contactos con los Shipibo, y los misioneros protestantes han seguido esta política con éxito, lo cual observé durante una jornada de campo en 1983-84.

\section{METODOS}

Los datos presentados aquí fueron recogidos en entrevistas universales en todos los hogares en ocho comunidades Shipibo ubicados en la ribera de los ríos Ucayali y Pisqui durante un plazo de 14 meses en 1983 y 1984 . Conseguíuna historia reproductiva completa de cada mujer de 13 años o más de edad ( $N=$ 386). Se identificó cada evento de nacimiento y la duración del intervalo entre los nacimientos con la mayor precisión posible. Las edades, duraciones de casamientos, y secuencias de nacimientos, y controles independientes para la precisión fueron obtenidos por estimaciones paralelas para hermanas y otros familiares, inspección departidas denacimiento, y consultageneral con miembros de la familia y vecinos.

Las definiciones de los efectos de poliginia fueron construidos:

i) una mujer estaba clasificada según si había estado en una unión polígina durante su vida;

ii) un determinado intervalo entre nacimientos fue clasificado según había ocurrido dentro del contexto de una unión polígina;

iii) la proporción de todos los intervalos cerrados entre los nacimientos fue calculada para cada mujer.

Adicionalmente, la duración promedio de los intervalos cerrados entre nacimientos fue calculada para cada mujer.

\section{RESULTADOS}

De un total de 1,445 personas enumeradas en censos de jure de ocho comunidades Shipibo, más de un tercio (885) estaban ubicadas en Paoyhän (Tabla 1). El ratio sexual del población total era 104 hombres por 100 mujeres, 
con mucha variación entre las comunidades. La Tabla 2 muestra una población muy joven con casi la mitad ( 49.3 por ciento) con menos de 15 años de edad, y 60.3 por ciento con menos de 20 años de edad.

Las tasas brutas de nacimiento para el año del censo tenían un rango de 42.6 hasta 89.6 por 1,000 . La mortalidad infantil global fue de 138 por 1,000. Las tasas de fecundidad global, total, y reproducción global tenían mucha variación (Tabla 4). El promedio de fecundidad completa era más bajo en la comunidad del Pisqui de 9 de octubre, que tenía una de las tasas más bajas de poliginia, y la tasa de fecundidad completa promedio más alta se observó en Irazola, donde la tasa de poliginia era una de las más baja.

La edad media de menarche $(N=307)$ era 13 , y la edad media del primer casamiento $(\mathrm{N}=271)$ era 14 años (Tablas 5,6$)$.

No había diferencia en la edad promedio de menarche por estado de poliginia (13.2 años de las monógamas por comparación con 13.1 de las políginas; $\mathrm{P}=0.144$ ), pero las mujeres políginas tenían una tendencia a casarse un año más temprano (14.1 años en comparación a 13.2) que las mujeres monógamas $(P=0.001)$. La edad promedio del primer parto para todas las mujeres parturientas con historias reproductivas completas $(N=237)$ fue 15.6 años, con un mediano de 15 y 14 años. La edad promedio del primer parto fue más baja para las mujeres políginas ( 15 años) que para las mujeres monógamas (15.8 años; $\mathrm{P}<0.01$ ).

La edad promedio de las 386 mujeres de 13 años o más que ya pasaron el menarche y de quienes he recogido historias reproductivas fue 30 años, edad mediana 27, y edad modal 18; la paridad promedio y mediana en este grupo fue cuatro. Cincuentidos mujeres estaban embarazadas en el momento de la entrevista. Solamente una de las 56 mujeres menopáusicas nunca ha tenido un embarazo.

De las mujeres de 15 años o más, 84.5 por ciento han experimentado por lo menos un embarazo, 85.6 por ciento se han casado, y 82.5 por ciento han experimentado por lo menos un parto (Tabla 7). Las edades promedios y medianas del último parto fueron 28.8 y 28.5 , respectivamente, y la duración promedio de reproducción para todas las mujeres parturientas fue 13 años. El intervalo promedio entre los nacimientos para todas las mujeres fue 31.5 meses con un mediano de 28.5 meses. Los intervalos promedios y medianos entre los nacimientos para las mujeres de 45 años o más $(\mathrm{N}=42)$ fueron 36.2 y 31.6 meses, respectivamente. 


\section{TABLA I Población por Comunidad}

$\begin{array}{lr}\text { Charashmanan } & 203 \\ \text { Vencedor } & 114 \\ \text { Tupac Amaru } & 111 \\ \text { Irazola } & 84 \\ \text { Santa Rosa } & 127 \\ \text { 9 De Octubre } & 48 \\ \text { Paococha } & 173 \\ \text { Paoyhan } & 585 \\ \text { Total } & 1,445\end{array}$

TABLA II Población por Edad y Sexo

Hombres Mujeres

Total

Edad Nurnero Por Ciento Numero Por Ciento Numero Por Ciento

\begin{tabular}{rrrrrrr}
\hline $0-4$ & 152 & 20.6 & 136 & 19.2 & 288 & 19.9 \\
$5-9$ & 114 & 15.5 & 120 & 16.9 & 234 & 16.2 \\
$10-14$ & 94 & 12.8 & 97 & 13.7 & 191 & 13.2 \\
$15-19$ & 82 & 11.1 & 77 & 10.9 & 159 & 11.0 \\
$20-24$ & 68 & 9.2 & 61 & 8.6 & 129 & 8.9 \\
$25-29$ & 43 & 5.8 & 45 & 6.4 & 88 & 6.1 \\
$30-34$ & 46 & 6.2 & 38 & 5.4 & 84 & 5.8 \\
$35-39$ & 30 & 4.1 & 42 & 5.9 & 72 & 5.0 \\
$40-44$ & 33 & 4.5 & 36 & 5.1 & 69 & 4.8 \\
$45-49$ & 21 & 2.8 & 11 & 1.6 & 32 & 2.2 \\
$50-54$ & 19 & 2.6 & 16 & 2.3 & 35 & 2.4 \\
$55-59$ & 14 & 1.9 & 9 & 1.3 & 23 & 1.6 \\
$60-64$ & 7 & 0.9 & 13 & 1.8 & 20 & 0.7 \\
$65-69$ & 6 & 0.8 & 4 & 0.6 & 10 & 0.7 \\
$70+$ & 8 & 1.1 & 3 & 0.4 & 11 & 0.8 \\
Total & 737 & 100.0 & 708 & 100.0 & 1445 & 100.0 \\
\hline
\end{tabular}


Con 1,274 intervalos entre nacimientos para todas las mujeres con 2 partos a término o más, el intervalo promedio entre nacimientos fue 31.0 meses con un mediano de 26.0 meses.

El análisis de regresión del orden de intervalo entre los nacimientos por duración de intervalo no muestra ningún aumento en la duración con el número de orden (Tabla 8) $B=0.0442, r^{2}$ ajustado $=0.0012 ; F=2.49 ; P=0.115$ ). Un estudio similar de edad al inicio de cada intervalo entre los nacimientos con la duración de intervalos muestra poca correlación $\left(B=0.032 ; r^{2}=0.001\right)$. Los intervalos entre los nacimientos no aumentan a medida que las mijeres envejecen.

Una tasa de fecundidad individual (TFI) fue calculada para cada mujer dividiendo su paridad por su trayecto reproductivo en años y multiplicando por 100 (Hern, 1990). El trayecto reproductivo es definido como el intervalo entre el primer partu a término o parto prematuro y el último, medido en meses y dividido por 12. El TFI promedio fue 56.8 con un mediano de 49.1.

De las 386 mujeres de 13 años o más, 75 (19.4 por ciento) han participado alguna vezen una reunión polígína. Cincuenta deellas, incluyendo algunas que tenían menos de 15 o más de 45 años de edad (13.0 por ciento del total) estaba en uniones políginas en el momento de la entrevista. Cuarenticinco de las mujeres de edad reproductiva (15-44) (o 15.7 por ciento) estában actualmente en uniones políginas; se hallaron las proporciones más altas en las comunidades del río Pisqui de 9 de octubre (45.5 por ciento), Vencedor, Tupac Amaru, y Charashmanan. La prevalencia más alta de intervalos políginos entre los nacimientos fue 56.6 por ciento y el más bajo fue 5.3 por ciento.

La proporción de hombres con edad de 15 años o más en uniones políginas fue 9.3 por ciento ( 16.6 por ciento de todos los hogares) con rango de 3.4 por ciento (Paoyhän) a 21.9 por ciento (Vencedor) (Tabla 9.)

\section{El efecto de la poliginía en la duración de intervalos entre los naci- mientos y la fecundidad individual}

Un hallazgo importante es que los intervalos promedios entre los nacimientos para las mujeres políginas tenían cuatro meses más de duración que los de las mujeres monógamas $(\mathbb{P}<0.02$; Tabla 10). Los $T F I$ promedios fueron significativamente más bajos para las mujeres políginas, que tenían 1.3 menos hijos por trayecto reproductivo que las mujeres monógamas ( $\mathrm{P}<0.001$, probabilidad de 2 causas: Tabla 11). El porcentage de niños vivos fue más alto para 
las mujeres monógamas que las mujeres políginas (70.9 por ciento comparado con 63.2 por ciento), pero esta diferencia puede ser atribuida a las edades relativamente más avanzadas de las mujeres políginas, con edad promedio de 40.4 comparado con el 29.8 para las mujeres monógamas.

La regresión de la proporción de los intervalos políginos entre nacimientos en el TFI rinde $B=-0.25067$ y un $r^{2}=0.05885, F=15.8(P=0.0001)$. Esta correlación negativa mínimo confirma el efecto supresivo de la poliginía en la fecundidad.

Al nivel dela comunidad, la fuerte correlación negativa entre la proporción cumulativa de los intervalos políginos entre los nacimientos y una variable de fecundidad es con la tasa general de fecundidad (Tabla 12). Hay una fuerte correlación negativa entre la poliginia y la fecundidad $(B=-0.84515)$, con un $r^{2}$ ajustado de 0.6667 ( $F=15 ; P<0.01 ;$ Tabla 13$)$.

La demostración más clara y fuerte de la relación entre la poliginía y la fecundidad se muestra en una regresión de la tasa general de fecundidad en la proporción cumulativa de intervalos políginos entre los nacimientos para las comunidades del río Pisqui solamente en el cual una línea casi derecha de correlación negativa es diagramada $(B=-0.96058)$ y $\mathrm{r}^{2}$ es $0.92271(\mathrm{P}<0.01)$. El misıno análisis con todas las comunidades menos Paoyhän rinde un resultado fuertemente semejante $(B=0.94786)$. Los resultados de esta sección muestran que:

* hay diferencias significativas en las duraciones de intervalos entre los nacimientos entre las mujeres políginas y las mujeres monógamas, con intervalos entre nacimientos para las mujeres políginas siendo un poco más de cuatro meses más prolongados;

* la fecundidad individual ès más baja para las mujeres políginas, que tienen un promediode 4.7 nacimientos por trayecto reproductivo en comparación con las mujeres monógamas, que tienen un promedio de 6.0 nacimientos por trayecto reproductivo;

* el análisis de regresión muestra una asociación positiva que es débil pero estadísticamente significativo entre la poliginia y las duraciones promedios de intervalos entre los nacimientos; 
TABLA III Tasas Vitales, por Comunidad

\begin{tabular}{lcccc}
\hline COMUNIDAD & $\begin{array}{c}\text { Población } \\
\text { Promedio } \\
\text { Durante ano }\end{array}$ & $\begin{array}{c}\text { Tasa Bruta } \\
\text { de }\end{array}$ & $\begin{array}{c}\text { Tasa Bruta } \\
\text { de } \\
\text { Natalidad }\end{array}$ & $\begin{array}{c}\text { Tasa de Aumento } \\
\text { Intrinsico }\end{array}$ \\
\hline Charashmanan & 201.5 & 59.6 & 44.7 & 14.9 \\
Vencedor & 112.5 & 44.4 & 17.8 & 26.2 \\
Tupac Amaru & 109.5 & 45.7 & 43.8 & 1.9 \\
Irazola & 83.0 & 84.3 & 60.2 & 24.1 \\
Santa Rosa & 124.0 & 80.7 & 32.3 & 48.4 \\
9 de Octubre & 48.5 & 42.6 & 63.8 & -21.2 \\
Paococha & 167.5 & 89.6 & 23.9 & 65.7 \\
Paoyhan & 572.5 & 57.6 & 14.0 & 43.6 \\
Todos & 1419.0 & 62.7 & 26.1 & 36.6 \\
\hline
\end{tabular}

TABLA IV Tasas de Fecundidad por Comunidad

\begin{tabular}{lccccc}
\hline COMUNIDAD & $\begin{array}{c}\text { Tasa General } \\
\text { de } \\
\text { Fecundidad }\end{array}$ & $\begin{array}{c}\text { Ratio de } \\
\text { Mujeres } \\
\text { a Niños** }\end{array}$ & $\begin{array}{c}\text { Tasa Global } \\
\text { de } \\
\text { Fecundidad }\end{array}$ & $\begin{array}{c}\text { Tasa Bruta } \\
\text { de }\end{array}$ & $\begin{array}{c}\text { Fecundidad } \\
\text { Rempletado }\end{array}$ \\
\hline Charashmanan & 0.255 & 0.787 & 8.355 & 3.805 & 7.6 \\
Vencedor & 0.148 & 0.926 & 7.715 & 6.000 & 9.0 \\
Tupac Amaru & 0.217 & 0.826 & 5.865 & 2.665 & 6.8 \\
Irazola & 0.353 & 0.941 & 10.835 & 5.415 & 10.0 \\
Santa Rosa & 0.400 & 1.160 & 7.265 & 4.095 & 8.4 \\
9 de Octubre & 0.182 & 0.546 & 2.500 & 1.250 & 6.0 \\
Paococha & 0.378 & 1.108 & 13.835 & 8.750 & 8.0 \\
Paoyhan & 0.271 & 0.943 & 8.145 & 4.385 & 6.9 \\
Todos & 0.278 & 0.932 & 8.467 & 4.379 & 7.6 \\
\hline
\end{tabular}

* Niños de edad 0-4 por mujer de edad 15-49

- Suma de tasas de fecundidad edad-especifica 
* el análisis de regresión muestra un efecto represivo de la poliginia en la fecundidad individual;

* hay una fuerte relación inequívocamente negativa entre la prevalencia comunitaria de poliginia y la fecundidad comunitaria que es demostrable en varias maneras y que constituye el resultado principal de este estudio.

\section{DISCUSION}

Varios hechos importantes surgen de esta revisión y estudio:

* Las estructuras políginas de la familia de algún tipo han sido observadas en los Shipibo desde el contacto más temprano con ellos;

* la poliginia tiene tanta importancia en los Shipibo que estaban animados a causar represalias violentas contra los que desalentaban esta costumbre (Steward y Metraux, 1948);

* los Shipibo comparten esta característica de estructura social con una gran variedad de sociedades indígenas de la Amazonía, algunas, otras agrupaciones panos, con enlaces lingüísticos con los Shipibo;

* la poliginia es ligada casi globalmente con la abstinencia sexual después del parto, con amenorrea de lactancia y con intervalos prolongados entre los nacimientos;

* los intervalos prolongados entre los nacimientos están estrechamente y casualmente asociados con la baja fecundidad en agrupaciones pre-industriales, con la sobrevivencia aumentada de niños, y con la mortalidad materna más baja (Yerushalmy, 1945; Wolfers y Scrimshaw, 1975; De Sweemer, 1984);

* la poliginia como estructura familiar no está aumentada en ninguna parte del mundo, incluyendo a los Shipibo - al contrario, su disminución y desaparación son evidencia del cambio cultural y además, de la "modernización" (Caldwell, 1982);

* la fecundidad de los Shipibo, a ambos niveles dela comunidad y la etnia, parece ser extremadamente alta. 
La diferencia entre las duraciones promedios de los intervalos entre los nacimientos de las mujeres políginas y las mujeres monógamas solamente es cuatro meses. Potter, et al (1963) señalaron que los intervalos promedios entre los nacimientos en exceso de 30 meses reflejan una amenorrea postpartum de duración promedio de casi un año, y ofrece evidencia presuntiva de una lactancia prolongada. Esta diferencia de cuatro meses puede explicar las diferenciasentrela fecundidad delas mujeres políginas ylas mujeres monógamas. Bongaarts ha mostrado que hasta una disminución moderada en la duración del prríodo post-partum de insusceptibilidad de nueve meses puede producir aumentos de más del 27 por ciento en la fecundidad marital para los Yoruba (Bongaarts, 1981). Las mujeres Shipibo amamantan a sus niños hasta uno o tres anios de edad, pero con intensidad variable. Los infantes Shipibo son llevados en la cadera y mantenidos allí hasta que casi pueden andar, y durante esta época maman en el momento que quieren. No observé ninguna diferencia entre las pıácticas de lactancia de las mujeres monógamas y las mujeres políginas.

Con los Shipibo, además, la poliginia tiene una correlación positiva con los intervalos prolongados entre los nacimientos, y tiene una correlación negativa con la fecundidad en todos sus aspectos. La fecundidad no está especialmente asociada con la edad del primer parto, y los intervalos entre los nacimientos no son afectados por la edad de la mujer, el número del intervalo, ni el orden del nacimiento (Bean y Mineau, 1986). Las duraciones promedios de los intervalos entre los nacimientos no son afectados por la ubicación de la comunidad.

Todas las medidas comunitarias y cumulativas de fecundidad individual son influidas por la prevalencia de la poliginia en la comunidad, pero la tasa general de fecundidad al instante es la medida más generả y se exhibe la asociación negativa más obvia y estadísticamente significativa con el índice cumulativo comunitario con la proporción de los intervalos entrelos nacimientos.

Si cualquier predicción puede ser hecha en base a este estudio, debe ser que los aumentos principales en la fecundidad para la mayoría de las comunidades Shipibo estudiadas aquí podrán ser observadas en el futuro. 
TABLA V Distribución por Frecuencia de Edades al Menarche, por Estado Polígino

Monógamo Polígíno Total

Edad Número Por ciento Número Por ciento Número Por ciento

\begin{tabular}{rrrrrrrr}
\hline 11 & 3 & 1.2 & 1 & 1.6 & 4 & 1.3 \\
& 12 & 44 & 18.0 & 12 & 19.4 & 56 & 18.2 \\
& 13 & 117 & 47.8 & 35 & 56.5 & 152 & 49.5 \\
& 14 & 57 & 23.3 & 11 & 17.7 & 68 & 22.1 \\
& 15 & 21 & 8.6 & 3 & 4.8 & 24 & 7.8 \\
& 16 & 3 & 1.2 & 0 & 0.0 & 3 & 1.0 \\
Total & & 245 & 100.0 & 62 & 100.0 & 307 & 100.0 \\
\hline
\end{tabular}

TABLA VI Distribución por Frecuencia, Edad de Primer Casamiento, por Estado Polígino

\begin{tabular}{rrrrrrr}
\hline Edad & $\begin{array}{c}\text { Monógamo } \\
\text { Número Por ciento }\end{array}$ & \multicolumn{2}{c}{$\begin{array}{c}\text { Polígino } \\
\text { Número Por ciento }\end{array}$} & $\begin{array}{c}\text { Total } \\
\text { Número Por ciento }\end{array}$ \\
\hline 8 & 0 & $0.0 \%$ & 1 & $.4 \%$ & 1 & $.4 \%$ \\
9 & 2 & $.7 \%$ & 0 & $0.0 \%$ & 2 & $.7 \%$ \\
10 & 1 & $.4 \%$ & 3 & $1.1 \%$ & 4 & $1.5 \%$ \\
11 & 6 & $2.2 \%$ & 2 & $.7 \%$ & 8 & $3.0 \%$ \\
12 & 24 & $8.9 \%$ & 11 & $4.1 \%$ & 35 & $12.9 \%$ \\
13 & 61 & $22.5 \%$ & 21 & $7.7 \%$ & 82 & $30.3 \%$ \\
14 & 42 & $15.5 \%$ & 12 & $4.4 \%$ & 54 & $19.9 \%$ \\
15 & 32 & $11.8 \%$ & 7 & $2.6 \%$ & 39 & $14.4 \%$ \\
16 & 238 & $.5 \%$ & 4 & $1.5 \%$ & 27 & $10.0 \%$ \\
17 & 72 & $.6 \%$ & 0 & $0.0 \%$ & 7 & $2.6 \%$ \\
18 & 8 & $3.0 \%$ & 0 & $0.0 \%$ & 8 & $3.0 \%$ \\
19 & 1 & $.4 \%$ & 0 & $0.0 \%$ & 1 & $.4 \%$ \\
20 & 2 & $.7 \%$ & 0 & $0.0 \%$ & 2 & $.7 \%$ \\
23 & 1 & $.4 \%$ & 0 & $0.0 \%$ & 1 & $.4 \%$ \\
Total & 210 & $77.5 \%$ & 61 & $22.5 \%$ & 271 & $100.0 \%$ \\
\hline
\end{tabular}


TABLA VII Distribución por Frecuencia del Número de Partos, Todas Las Mujeres de Edad 15+

\begin{tabular}{cccc}
\hline Número & Mujeres & Por ciento \\
& 0 & 62 & 17.5 \\
1 & 31 & 8.8 \\
2 & 26 & 7.3 \\
3 & 37 & 10.5 \\
4 & 30 & 8.5 \\
5 & 32 & 9.0 \\
6 & 18 & 5.1 \\
7 & 37 & 10.5 \\
8 & 19 & 5.4 \\
9 & 20 & 5.6 \\
Total & 10 & 20 & 5.6 \\
& 11 & 17 & 4.8 \\
& 12 & 3 & 0.8 \\
& 13 & 2 & 0.6 \\
\hline
\end{tabular}

TABLA VIII Duración de Intervalos entre los Nacimientos por Número de Intervalo

\begin{tabular}{ccccc}
\hline $\begin{array}{c}\text { Número de } \\
\text { Intervalo }\end{array}$ & Promedio & $\begin{array}{c}\text { Patrón de } \\
\text { Mediano }\end{array}$ & Dispersión & N \\
\hline 1 & 31 & 24 & 24 & 248 \\
2 & 31 & 25 & 16 & 225 \\
3 & 30 & 27 & 15 & 187 \\
4 & 32 & 28 & 21 & 158 \\
5 & 28 & 26 & 13 & 132 \\
6 & 33 & 29 & 19 & 111 \\
7 & 34 & 30 & 18 & 81 \\
8 & 30 & 27 & 13 & 60 \\
9 & 35 & 29 & 19 & 42 \\
10 & 36 & 32 & 19 & 21 \\
11 & 30 & 26 & 12 & 6 \\
12 & 26 & 26 & -- & 2 \\
\hline
\end{tabular}


TABLA IX Proporción de Hombres de Edad 15+ en Uniones Políginas, por Comunidad

\begin{tabular}{lccccc}
\hline Comunidad & $\begin{array}{c}\text { \# de Hogares } \\
\text { por Soltero }\end{array}$ & Monógamo & $\begin{array}{c}\text { Poligino } \\
\text { Número }\end{array}$ & $\begin{array}{l}\text { Total } \\
\text { Por ciento }\end{array}$ & \\
\hline Charashmanan & 25 & 47 & 9 & $(16.7 \%)$ & 56 \\
Vencedor & 14 & 25 & 7 & $(21.9 \%)$ & 32 \\
Tupac Amaru & 18 & 28 & 4 & $(12.5 \%)$ & 32 \\
Irazola & 13 & 23 & 1 & $(4.2 \%)$ & 24 \\
Santa Rosa & 16 & 33 & 2 & $(5.7 \%)$ & 35 \\
9 de Octubre & 8 & 12 & 2 & $(14.3 \%)$ & 14 \\
Paococha & 25 & 29 & 5 & $(14.7 \%)$ & 34 \\
Paoyhan & 92 & 14 & 35 & $(3.4 \%)$ & 148 \\
\hline Total & 211 & 340 & 35 & $(9.3 \%)$ & 375
\end{tabular}

TABLA X Duración Promedio de Intervalos entre los Nacimientos, por Estado Polígino

\begin{tabular}{lccc}
\hline ESTADO & Promedio & Mediano & N \\
\hline Polígíno & 34.5 & 31.4 & 68 \\
Monógamo & 30.3 & 27.7 & 167 \\
Total & 31.5 & 28.5 & 235 \\
\hline
\end{tabular}




\section{TABLA XI Tasa Individual de Fecundidad,} por Estado Polígino

\begin{tabular}{|c|c|c|c|}
\hline ESTADO & Promedio & Mediano & $\mathrm{N}$ \\
\hline Polígíno & 47.09 & 44.44 & 68 \\
\hline Monógamo & 60.64 & 51.53 & 167 \\
\hline Total & 56.75 & 49.12 & 235 \\
\hline \multicolumn{4}{|c|}{$\begin{array}{l}\text { TABLA XII Tasa General de Fecundidad y } \\
\text { Proporción de Intervalos Políginos entre } \\
\text { los Nacimientos, por Comunidad }\end{array}$} \\
\hline Comunidad & & $\begin{array}{l}\text { eneral } \\
\text { didad }\end{array}$ & $\begin{array}{l}\text { Proporción de Intervalos } \\
\text { Políginos Entre los } \\
\text { Nacimientos }\end{array}$ \\
\hline Charashmanan & & 255. & 0.433 \\
\hline Vencedor & & 148 & 0.523 \\
\hline Tupac Amaru & & 217 & 0.455 \\
\hline Irazola & & 353 & 0.067 \\
\hline Santa Rosa & & 100 & 0.080 \\
\hline 9 de Octubre & & 182 & 0.500 \\
\hline Paococha & & 378 & 0.201 \\
\hline Paoyhan & & 271 & 0.070 \\
\hline Todas Comunidades & & 278 & 0.224 \\
\hline
\end{tabular}


TABLA XIII Analisis de Regresión del Proporción de Intervalos entre los Nacimientos en la Tasa General de Fecundidad, para Ocho Comunidades de los Shipibo

R Múltiple

R Cuadrado

R Cuadrado Ajustado

Patrón de Error
.84515

.71428

.66666

.05384

Análisis de Variación

DF Suma de los Cuadrados

Regresión

Residual

1

.04348

.01739

Cuadrado Promedio

.04348

.00290

$F=14.99944$

Signif $F=.0082$

Ecuación Número 1 Variable Dependente.. GFR

$\begin{array}{lccccc}\text { Variable } & \text { B } & \text { SE B } & \text { Beta } & \text { T } & \text { Sig T } \\ \text { PROPOLY } & -.38314 & .09893 & -.84515 & -3.873 & .0082 \\ \text { (constante) } & .38704 & .03452 & & 11.211 & .0000\end{array}$




\section{CONCLUSIONES}

Parece que, para las comunidades estudiadas:

* la poliginia está asociada con la fecundidad individual más baja;

* la modernización resulta en una prevalencia comunitaria de poliginia disminuida;

* la modernización no parece estar asociada con la fecundidad más baja, sino con la más alta, en los Shipibo.

Estos resultados apoyan la hipótisis general de que la disrupción de las prácticas culturales tradicionales que mantienen la fecundidad a niveles bajos contribuyen a una fecundidad no solamente más alta sino hasta no-controlada. Los resultados deben ser interpretados con cuidado porque no es posible determinar hasta qué punto los resultados representan a todos los Shipibo u otras poblaciones indígenas en general. Hasta el punto que representan, estos datos apoyan una conclusión inportante: que la fecundidad probablemente va a aumentar en las sociedades que experimentan cambio cultural muy rapido en sociedades tribales hasta campesinos y urbanos.

* Este artículo ha sido publicado en una primera versión en inglés bajo el mismo título en la Revista Population Studies, 46:53-64, 1992. Esta versión en castellano está a cargo del mismo autor 


\section{BIBLIOGRAFIA}

\section{ABELOVE, J.M.}

1978 Pre-Verbal Learning of Kinship Behavior Among Shipibo Infants of Eastern Peru, Doctoral dissertation, City University of New York.

\section{ABORAMPAHO.}

1987 Plural marriage and fertility differentials: A study of the Yoruba of Western Nigeria. Human Organization 46:29-38.

ANDERTON, D.L. and R.J. EMIGH

1989 Polygynous fertility: sexual competition versus progeny. American Journal of Sociology 94:832-855.

BEAN, L.L. and G.P. MINEAU

1986 The polygyny-fertility hypothesis: a re-evaluation. Population Studies 40:67-81.

BEHRENS, CLIFFORD A.

1984 Shipibo Ecology and Economy. Doctoral Dissertation in Anthropology, UCLA.

1989 Scientific basis for Shipibo soil classifications and land use: changes in soil-plant associations with cash cropping. American Anthropologist 91:83-100.

BERGMAN, ROLAND W.

1980 Amazon Economics: The Simplicity of Shipibo Indian Wealth. Ann Arbor: University Microfilms International.

BONGAARTS, JOHN

1978 A framework for analyzing the proximate determinants of fertility. Population and Development Review 4:105-132.

1981 The impact on fertility of traditional and changing child-spacing practices. In Child-Spacing in Tropical Africa, H.J. Page and R Lesthaeghe, eds. London: Academic Press. p 111.

BONGAARTS, J. and R.G.' POTTER

1983 Fertility, Biology, and Behavior: An Analysis of the Proximate Determinants. New York:Academic Press. 
BORGERHOFF MULDER, $M$.

1989 Marital status and reproductive performance in Kipsigis women: Reevaluating the polygyny-fertility hypothesis. Population Studies 43:285304.

BOWERS, NANCY.

1971 Demographic problems in montane New Guinea. In: Culture and Population: A Collection of Current Studies, S. Polgar, ed. Chapel Hill: Carolina Population Center.

CALDWELL, J.C.

1982 Theory of Fertility Decline. New York: Academic Press. p 289.

CALDWELL, J.C., and CALDWELL, PAT

1977 The role of marital sexual abstinence in determining fertility: a study of the Yoruba in Nigeria. Population Studies 31:193-217.

CALDWELL, J., P. CALDWELL, and B. CALDWELL.

1987 The function of child-spacing in traditional societies and the direction of change. Current Anthropology 28:25-43.

CHAGNON, NAPOLEON A.

1977 Yanomamo: The Fierce People. Second Edition. New York: Holt, Rinehart, and Winston.

1979 Is reproductive success equal in egalitarian societies? In: Chagnon NA, Irons W (eds): Evolutionary Biology and Human Social Behavior: An Anthropological Perspective. North Scituate, MA: Duxbury Press.

CHOINACKA, HELENA

1980 Polygyny and the rate of population growth. Population Studies 34:91107.

CLEVELAND, D.A.

1987 The political economy of fertility regulation: The Kusasi of Svanna West Africa (Ghana). In Culture and Reproduction, W.P. Handwerker, ed. Boulder: Westview Press. pp 283-284

DE SWEENER, C.

1984 The influence of child spacing on child survival. Population Studies $38: 47-72$. 
DORJAHN, VERNON R.

1958 Fertility, polygyny, and their interrelations in Temne society. American Anthropologist 60:838-860.

EARLY, J.D. and J.F. PETERS

1990 The Population Dynamics of the Mucajai Yanomama. San Diego, Cal.:Academic Press.

GARENNE, M. and E. VAN DE WALLE.

1989 Polygyny and fertility among the Sereer of Senegal. Population Studies 43:267-283.

GREENLAND, $\mathrm{S}$. and NEUTRA, $R$.

1980 Control of confounding in the assessment of medical technology. International Journal of Epidemiology 9:361-367.

HANDWERKER, W.P.

1987 "Natural fertility" as a balance of choice and behavioral effect: policy implications for Liberian farm households. In Culture and Reproduction, W.P.Handwerker, ed. p 107-108

HERN, WARREN M.

1976 Knowledge and use of herbal contraceptives in a Peruvian Amazon village. Human Organization 35:9-19.

1977 High fertility in a Peruvian Amazon Indian village. Human Ecology 5:355-368.

1988 Polygyny and Fertility Among the Shipibo: An Epidemiologic Test of An Ethnographic Hypothesis, Unpublished Ph.D. dissertation, University of North Carolina School of Public Health.

1990 Individual fertility rate: A new individual fertility measure for small populations. Social Biology 37:102-109.

ISAAC, BARRY L.

1980 Female fertility and marital form among the Mende of rural Upper Bambara Chiefdom, Sierra Leone. Ethnology 19:297-313. 
JACKSON, J.

1983 The Fish People: Linguistic Exogamy and Tukanoan Identity in Northwest Amazonia. Cambridge, Mass: MIT Press

JOHNSTON, F.E., KENSINGER, K.M., JANTZ, R., et al.

1969 The population structure of the Peruvian Cashinahua: demographic, genetic, and cultural interrelationships. Human Biology 41:29-41.

JOHNSTON, F.E. and KENSINGER, K.M.

1971 Fertility and mortality differentials and their implications for microevolutionary change among the Cashinahua. Human Biology 43:356-364.

KONNER, M. and C. WORTHMAN

1980 Nursing frequency, gonadal function, and birth spacing among !Kung hunter-gatherers. Science 207:788

LEE, RICHARD B.

1980 Lactation, ovulation, infanticide, and women's work: a study of huntergatherer population regulation. In: Cohen MN, Malpass RS, Klein HG (eds.): Biosocial Mechanisms of Population Regulation. New Haven: Yale UUniversity Press.

NAG, MONI

1980 How modernization can also increase fertility. Current Anthropology 21:571-587.

NEEL, J.V. and CHAGNON, N.A.

1968 The demography of two tribes of primitive, relatively unacculturated American Indians. Proceedings of the National Academy of Sciences 59:680-689.

NEEL, J.V. and SALZANO, F.M.

1970 Further studies on the Xavante Indians. X. Some hypothesesgeneralizations resulting from these studies. American Journal of Human Genetics 19:554-574.

OLUSANYA, P.O.

1971 The problem of multiple causation in population analysis, with particular reference to the polygamy-fertility hypothesis: Sociological Review 19:165-178. 
PAGE, H.J. and R. LESTHAEGHE, (Eds.)

1981 Child-Spacing in Tropical Africa London:Academic Press.

PETERSEN, W.

19.75 A demographer's view of prehistoric demography. Current Anthropology 16:227-245.

POTTER, R.G., WYON, J.B., PARKER, M., et al

1963 A case study of birth interval dynamics. Population Studies 17:155-166.

Schoenmaeckers, R., I.H. Shah, et al.

1981 The child-spacing tradition and the postpartum taboo in tropical Africa: anthropological evidence. In Page and Lesthaeghe, op.cit." p 25.

SHAIKH, K., K.M.A. AZIZ, and A.I. CHOWDHURY

1987 Differentials of fertility between polygynous and monogamous marriages in rural Bangladesh. Journal of Biosocial Science 19:49-56.

SISKIND, JANET

1973 Tropical forest hunters and the economy of sex. In Peoples and Cultures of Native South America, by D.R. Gross (ed.). Garden City: The Natural History Press.

SMITH, J.E. and KUNZ, P.R.

1976 Polygyny and fertility in nineteenth-century America.Population Studies 30:465-480.

STEWARD, J.H. and METRAUX A.

1948 Tribes of the Peruvian and Ecuadorian Montan a. In Handbook of South American Indians, J.H. Steward, ed. Vol.3. p 51-59, 290-347, p555. The Tropical Forest Tribes,Bulletin 143. Washington, D.C: Bureau of Ethnology.

VAN ARSDALE, P.W.

1978 Population dynamics among Asmat hunter-gatherers of New Guinea: data, methods, comparisons. Human Ecology 6: 435-467. 
WHITING JOHN W.M.

1964 The effects of climate on certain cultural practices. In Explorations In Cultural Anthropology, WH Goodenough (ed.) p 511. New York: McGraw-Hill.

WOLFERS, D. and SCRIMSHAW, S.

1975 Child survival and intervals between pregnancies in Guayaquil, Ecuador. Population Studies 29:479-496.

WOOD, J.W., JOHNSON, P.L., and CAMPBELL, K.L.

1985 Demographic and endocrinological aspects of low natural fertility in highland New Guinea. Journal of Biosocial Science 17:57-79.

YERUSHALMY, J.

1945 On the interval between successive births and its effect on survival of infant. Human Biology 17:65-106. 